\title{
Inibição de diferentes formas de ocratoxina derivadas de Aspergillus carbonarius ao empregar Bacillus spp. como estratégia de biocontrole em uvas
}

\author{
Rafaela Diogo Silveira ${ }^{1}$, Flávio Fonseca Veras ${ }^{1}$, Adriano Brandelli $^{1}$, Juliane Elisa Welke ${ }^{1}$
}

\begin{abstract}
Resumo
Espécies de Bacillus têm sido utilizadas como estratégia de biocontrole para reduzir o uso de fungicidas sintéticos na viticultura, os quais podem trazer diversas consequências negativas, incluindo o risco ocupacional e geração de resíduos no solo, rios e nas próprias uvas. Aspergillus carbonarius é um fungo encontrado em uvas que pode produzir ocratoxina A (OTA), entre outras ocratoxinas (OTs), em uvas. O objetivo deste trabalho foi avaliar a capacidade de quatro cepas de Bacillus spp. (P1, P7, P11 e P45) em inibir o desenvolvimento de $A$. carbonarius e a síntese de OTs em uvas. A ocorrência de formas de ocratoxina (OT) foi verificada com o uso da cromatografia líquida acoplada à espectrometria de massas com analisador híbrido quadrupolo-tempo de voo (LC-QtoF-MS). As uvas tratadas com a cepa P1 não apresentaram colônias fúngicas (100\% de redução), já as cepas P7, P11 e P45 causaram redução fúngica em 95, 95 e 61\%, respectivamente. Além da OTA, foram identificadas outras seis formas de OTs nas uvas inoculadas com $A$. carbonarius: OT $\alpha$, OT $\beta$, OT $\alpha$ metil-éster, OT $\alpha$ amida, e N-formil-OT a amida. Dessas, quatro OTs (OT $\beta$, OTa metil-éster, OTa amida, $\mathrm{N}$-formil-OTa amida) foram relatadas pela primeira vez em uvas. Todas as cepas de Bacillus testadas foram capazes de inibir a síntese de OTA. Nas uvas tratadas com as cepas P1 e P7 nenhuma OT foi encontrada. Já nas uvas tratadas com as cepas P11 e P45, OTa e OT $\beta$ foram detectadas. Nas uvas tratadas com a cepa P45 também identificamos OTa metil-éster e a N-formil-OTa amida.
\end{abstract}

Palavras-chave: biofungicida; ocratoxina A; Bacillus; uvas; ocratoxinas.

\section{Introdução}

Um dos desafios na viticultura é o controle fúngico, pois as condições climáticas das regiões vitivinícolas favorecem a ocorrência desses microrganismos. A ocorrência de Aspergillus carbonarius em uvas é menos frequente do que outros fungos do mesmo gênero, entretanto, quase todos os isolados produzem níveis altos de OTA (WELKE, 2019). A exposição a essa micotoxina está relacionada a efeitos genotóxicos, nefrotóxicos, teratogênicos, imunossupressores, entre outros (CIMBALO; FONT; MANYES, 2020). Além disso, a OTA está classificada como possivelmente carcinogênica para humanos (grupo 2B) pela Agência Internacional de Pesquisa sobre o Câncer (IARC, 1993). Além da OTA, outras OTs já foram identificadas em culturas de $A$. carbonarius, como a OTa, OT $\beta$, OTC, entre outras (GIL-SERNA; VÁZQUEZ; PATIÑO, 2020; WANG et al., 2016).

O uso de fungicidas sintéticos é uma prática comum para o controle das infecções fúngicas. Entretanto, sua aplicação está associada ao aumento da resistência dos fungos a esses produtos químicos, além de causar danos ao ambiente e à saúde humana (ZHANG, Hongyin et al., 2020). Devido ao impacto negativo causado pelo uso de fungicidas sintéticos, o estudo de métodos alternativos utilizando agentes de bioncotrole vem se destacando. Espécies de Bacillus têm sido exploradas por possuírem o potencial de inibir o desenvolvimento fúngico e a biossíntese de micotoxinas (REN et al., 2020). A atividade antifúngica de Bacillus spp. pode estar relacionada à capacidade de sintetizar lipopeptídeos, sideróforos, compostos voláteis e enzimas, além de competição por espaço e nutrientes com os patógenos (CALVO et al., 2019; CAULIER et al., 2019).

Em trabalho prévio, dez cepas de Bacillus isoladas de ambiente aquático na região Amazônica, Brasil, foram testadas contra fungos toxigênicos em meio de cultura (VERAS et al., 2016). O objetivo deste estudo foi avaliar as quatro cepas mais promissoras selecionadas como agentes de biocontrole para a inibição de $A$. carbonarius e a ocorrência de OTs em uvas.

\section{Material e Métodos}

Uvas (Vitis vinífera) cultivar Chardonnay, colhidas em 2019 na cidade de Canela, Rio Grande do Sul, Brasil, foram usadas. As uvas foram submetidas à desinfeção com hipoclorito de sódio $1 \%$.

A. carbonarius (ITAL293) foi cultivado em meio ágar batata dextrose (BDA) por 7 dias a $25^{\circ} \mathrm{C}$. As cepas de Bacillus utilizadas neste estudo (P1, P7, P11 e P45) foram isoladas de ambiente aquático da região Amazônica, e previamente descritas como as mais promissoras contra fungos toxigênicos em testes in vitro (VERAS et al., 2016). As cepas de Bacillus foram cultivadas em caldo Brain-Heart infusion $(\mathrm{BHI})$ à $37^{\circ} \mathrm{C}$.

\footnotetext{
${ }^{1}$ Instituto de Ciência e Tecnologia de Alimentos (ICTA), Universidade Federal do Rio Grande do Sul (UFRGS), Av. Bento Gonçalves, 9500, Prédio 43212, CEP 91501-970, Porto Alegre, RS, Brasil. e-mail: rafaela_dsilveira@hotmail.com
} 
O efeito das cepas de Bacillus (P1, P7, P11 e P45) sobre o crescimento de $A$. carbonarius em uvas foi avaliado através da imersão das bagas na suspensão de células de Bacillus $10^{9}$ UFC $\mathrm{mL}^{-1}$, e posteriormente na suspensão de esporos de $A$. carbonarius $10^{3}$ esporos $\mathrm{mL}^{-1}$. As uvas foram incubadas por 7 dias a $30^{\circ} \mathrm{C}$. O efeito inibitório das cepas de Bacillus foi avaliado pela contagem de colônias de fungos e por inspeção visual de acordo com JIANG et al., (2014).

As OTs foram extraídas das uvas com acetonitrila:ácido fórmico $0,1 \%(\mathrm{v} / \mathrm{v})$ e analisadas com o uso da LC-QTOF-MS em coluna $\mathrm{C}_{18}$, fase móvel composta por água MilliQ:ácido acético:acetato de amônia e acetonitrila:ácido acético:acetato de amônia com fluxo de 0,4 mL min.-1. Os compostos foram identificados através do tempo de retenção, massa protonada, padrão de fragmentação, e ordem de eluição na coluna C18 em comparação com os dados da literatura (BITTNER et al., 2015; GONZÁLEZ-ARIAS et al., 2017; ZHANG, Jian et al., 2016).

As análises estatísticas foram realizadas no software XLSTAT2017 (Addinsoft, New York, USA).

\section{Resultados e Discussão}

A contagem de colônias fúngicas verificada nas bagas de uvas inoculadas com as cepas de Bacillus e a porcentagem de bagas de uvas que não apresentaram desenvolvimento fúngico após o uso dos agentes de biocontrole está apresentada na Figura 1. Todas as cepas bacterianas causaram redução significativa ( $p<0,05$, de acordo com o teste $t$ de Student) na contagem de colônias fúngicas quando comparadas às uvas do grupo controle. As cepas P1, P7 e P11 foram as mais eficientes. As bagas tratadas com a cepa P1 não apresentaram colônias de fungos (100\% de redução), enquanto as cepas P7 e P11 causaram uma redução de cerca de $95 \%$ na contagem de fungos. A menor porcentagem de redução nas contagens de colônias de fungos foi encontrada para a cepa P45 (61\%).
A espessura e a dureza da casca da uva são fatores-chave na colonização de $A$. carbonarius. Cultivares com menor espessura de casca e dureza, como as uvas Chardonnay em estudo, são mais suscetíveis à colonização por fungos do que outras cultivares. Portanto, controlar o crescimento de $A$. carbonarius torna-se mais desafiador em cultivares com essas características de casca (VERAS et al., 2020).

A Tabela 1 mostra o tempo de retenção, massa monoisotópica e relação massa-carga $(\mathrm{m} / \mathrm{z})$ de íons precursores das OTs detectadas usando LC-QToFMS. Foram encontradas seis formas de OT nas uvas inoculadas com $A$. carbonarius, incluindo OTa, OT $\beta$, OTa metil-éster, OTa amida, N- formil- OTa amida e OTA. A presença de OT $\beta$, OT $\alpha$ metil-éster, OT $\alpha$ amida e N-formil-OTa amida não foi relatada anteriormente em uvas.

O efeito das diferentes cepas de Bacillus sobre a ocorrência de OTs está disposto na Figura 2. As OTs foram quantificadas como equivalente à OTA usando uma curva analítica $(\mathrm{y}=317673 \mathrm{x}$ 6255,1 ) com linearidade adequada (coeficiente de determinação, $R^{2}$ de 0,9998), limite de detecção (LOD; 0,01 $\mu \mathrm{g} \mathrm{kg}^{-1}$ ) e o limite de quantificação (LOQ; 0,05 $\mu \mathrm{g} \mathrm{kg}^{-1}$ ) mostram que o método é suficientemente sensível para quantificar OTs em uvas, uma vez que esses valores são, respectivamente, 200 e 40 vezes inferiores ao limite máximo permitido para OTA em produtos de uva $\left(2 \mu \mathrm{g} \mathrm{kg}^{-1}\right)$ pela Comissão Europeia (EUROPEAN COMMISSION (EFSA), 2006) e agência Brasileira (ANVISA, 2011). GALLO et al., (2012) relataram o OTa como produto da hidrólise da OTA por $A$. carbonarius, o que foi sugerido como estratégia de autoproteção. Esta hipótese também poderia ser investigada em relação ao metabolismo de $A$. carbonarius na presença de Bacillus spp. A inibição da síntese de OTA também foi verificada quando Bacillus vallismortis EBHVSH28 e $B$. amyloliquefaciens EBHVSH29 foram usados como estratégia de biocontrole para uvas. Outras ocratoxinas não foram avaliadas (EL-SHANSHOURY et al., 2018).

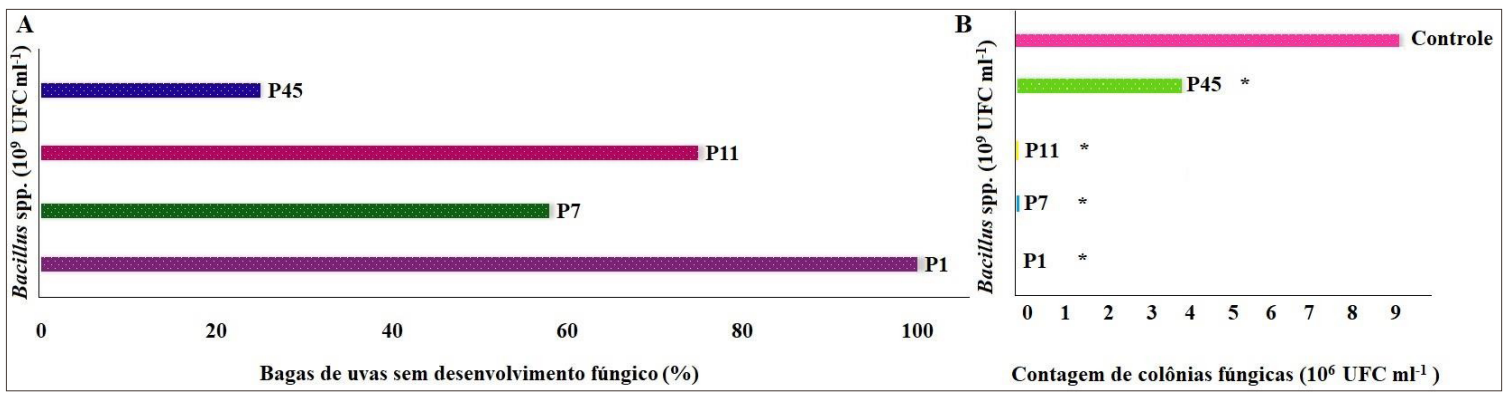

Figura 1: Porcentagem de bagas de uvas que não apresentaram desenvolvimento fúngico após o uso dos agentes de biocontrole (A) e contagem de colônias fúngicas verificadas nas bagas de uvas inoculadas com as cepas de Bacillus (B). O asterisco indica uma diferença significativa entre as uvas tratadas com Bacillus e as uvas controle $(p<0,05$, de acordo com o teste t de Student).

Tabela 1: OTs identificadas nas uvas inoculadas com A. carbonarius $\left(10^{3}\right.$ esporos $\left.\mathrm{mL}^{-1}\right)$

\begin{tabular}{|c|c|c|c|c|c|c|}
\hline Micotoxina & OTa & От $\beta$ & OTa metil- éster & OTa amida & $\begin{array}{c}\text { N-Formil- OTa } \\
\text { amida }\end{array}$ & OTA \\
\hline \multicolumn{7}{|l|}{ Variáveis analíticas } \\
\hline TR $\quad(\min )^{\mathrm{a}}$ & 1,0 & 1,1 & 1,2 & 1,4 & 1,5 & 2,5 \\
\hline Massa monoisotópica & 256,0139 & 222,0528 & 270,0295 & 255,0298 & 283,0247 & 403,0823 \\
\hline$m / z$ íon precursor e modo & $\begin{array}{c}294,9776 \\
{[\mathrm{M}+\mathrm{K}]^{+}}\end{array}$ & $\begin{array}{l}245,0426 \\
{[\mathrm{M}+\mathrm{Na}]^{+}}\end{array}$ & $\begin{array}{c}253,0268 \\
{[\mathrm{M}+\mathrm{H}-\mathrm{H} 2 \mathrm{O}]^{+}}\end{array}$ & $\begin{array}{c}273,0642 \\
{[\mathrm{M}+\mathrm{NH} 4]^{+}}\end{array}$ & $\begin{array}{c}284,0326 \\
{[\mathrm{M}+\mathrm{H}]^{+}}\end{array}$ & $\begin{array}{c}404,0901 \\
{[\mathrm{M}+\mathrm{H}]^{+}}\end{array}$ \\
\hline
\end{tabular}

a Tempo de retenção em minutos. 
Todas as cepas de Bacillus foram capazes de inibir a síntese de OTA, a forma mais tóxica de OT. As uvas tratadas com Bacillus sp. P1 não apresentaram crescimento fúngico, e consequentemente, nenhuma OT foi encontrada quando esta cepa foi usada nas uvas. Bacillus sp. P7 também foi capaz de inibir todas as OTs que foram identificadas nas uvas do grupo controle.

Nas uvas tratadas com Bacillus sp. P11, OTa e OT $\beta$ foram detectadas. Níveis semelhantes dessas OTs foram encontrados nas uvas tratadas com a cepa P45, além das outras formas de OTs como OTa metil-éster e a N-formil-OTa amida. Os níveis encontrados nas uvas tratadas com essas cepas (P11 e P45) foram cerca de $97 \%$ mais baixos do que aqueles encontrados nas uvas controle.

\section{Referências}

ANVISA. RDC No 7, de 18 de fevereiro de 2011. Regulamento Técnico sobre limites máximos tolerados para micotoxinas em alimentos. 2011.

BITTNER, Andrea; CRAMER, Benedikt; HARRER, Henning; HUMPF, Hans Ulrich. Structure elucidation and in vitro cytotoxicity of ochratoxin $\alpha$ amide, a new degradation product of ochratoxin $A$. Mycotoxin Research, v. 31, n. 2, p. 83-90, 2015.

CALVO, H.; MENDIARA, I.; ARIAS, E.; BLANCO, D.; VENTURINI, $M$. E. The role of iturin A from B. amyloliquefaciens BUZ-14 in the inhibition of the most common postharvest fruit rots. Food Microbiology, v. 82, n. January, p. 62-69, 2019.

CAULIER, Simon; NANNAN, Catherine; GILLIS, Annika; LICCIARDI, Florent; BRAGARD, Claude; MAHILLON, Jacques. Overview of the antimicrobial compounds produced by members of the Bacillus subtilis group. Frontiers in Microbiology, v. 10, n. FEB, p. 1-19, 2019.

CIMBALO, A; FONT, G; MANYES, L. Toxicity of mycotoxins in vivo on vertebrate organisms: A review. Food and Chemical Toxicology, v. 137, p. 111-161, 2020.

EL-SHANSHOURY, Abd El-Raheem R; EL-HALMOUCH, Yasser $\mathrm{H}$; MOHAMED, Samia F; FAREED, Mervat F. Potential of Grape Epiphytic Antagonists to Biocontrol Aspergillus Transmission and Accumulation of Aflatoxin B1 and Ochratoxin A in Post Harvest Taify Table Grape. Journal of Plant Pathology \& Microbiology, v. 9, n. 10, p. 1-10, 2018.

EUROPEAN COMMISSION (EFSA). Commission Regulation (EC) No 1881/2006 of 19 December 2006 setting maximum levels for certain contaminants in foodstuffs. Off J Eur Union, 2006.

GALLO, Antonia; BRUNO, Kenneth S.; SOLFRIZZO, Michele; PERRONE, Giancarlo; MULE, Giuseppina; VISCONTI, Angelo; BAKER, Scott E. New insight into the ochratoxin a biosynthetic pathway through deletion of a nonribosomal peptide synthetase gene in Aspergillus carbonarius. Applied and Environmental Microbiology, v. 78, n. 23, p. 8208-8218, 2012.

GIL-SERNA, Jéssica; VÁZQUEZ, Covadonga; PATIÑO, Belén. Genetic regulation of aflatoxin, ochratoxin A, trichothecene, and fumonisin biosynthesis: A review. International Microbiology, v. 23, n. 1, p. 89-96, 2020.

GONZÁLEZ-ARIAS, Cyndia A.; MARÍN, Sonia; ROJAS-GARCÍA, Aurora E.; SANCHIS, Vicente; RAMOS, Antonio J. UPLC-MS/ MS analysis of ochratoxin A metabolites produced by Caco-2 and HepG2 cells in a co-culture system. Food and Chemical Toxicology, v. 109, p. 333-340, 2017.

\section{Conclusões}

As cepas de Bacillus P1, P7, P11 e P45 mostraram potencial para serem utilizadas como agentes de controle biológico para prevenir ou reduzir o crescimento de $A$. carbonarius e a ocorrência de OTs em uvas. A síntese da OTA, a forma mais tóxica da OT, foi inibida pelas quatro cepas de Bacillus em estudo. A cepa P1 revelou ser o agente de biocontrole mais promissor, apresentando $100 \%$ de inibição fúngica. Seis formas de OT foram identificadas por LC-QToF-MS nas uvas inoculadas com A. carbonarius: OTa, OT $\beta$, OTa metil-éster, OTa amida, e $\mathrm{N}$-formil-OTa amida. O potencial promissor das cepas de em inibir o desenvolvimento de $A$. carbonarius e síntese de OTs em uvas abre perspectivas para novas opções de biofungicidas para a agricultura.

IARC. International Agency of Research on Cancer. Monographs on the Evaluation of Carcinogenic Risks to Humans. 1993.

Disponível em: https://monographs.iarc.who.int/agents-classifiedby-the-iarc.

JIANG, Chunmei; SHI, Junling; LIU, Yanlin; ZHU, Chengyong. Inhibition of Aspergillus carbonarius and fungal contamination in table grapes using Bacillus subtilis. Food Control, v. 35, n. 1, p. 41-48, 2014. DOI 10.1016/j.foodcont.2013.06.054.

REN, Xianfeng; ZHANG, Qi; ZHANG, Wen; MAO, Jin; LI, Peiwu. Control of aflatoxigenic molds by antagonistic microorganisms: Inhibitory behaviors, bioactive compounds, related mechanisms, and influencing factors. Toxins, v. 12, n. 1, p. 1-22, 2020.

VERAS, Flávio Fonseca; CORREA, Ana Paula Folmer; WELKE, Juliane Elisa; BRANDELLI, Adriano. Inhibition of mycotoxinproducing fungi by Bacillus strains isolated from fish intestines. International Journal of Food Microbiology, v. 238, p. 23-32, 2016.

VERAS, Flávio Fonseca; DACHERY, Bruna; MANFROI, Vitor; WELKE, Juliane Elisa. Colonization of Aspergillus carbonarius and accumulation of ochratoxin Aa in Vitis vinifera, Vitis labrusca, and hybrid grapes - research on the most promising alternatives for organic viticulture. Journal of the Science of Food and Agriculture, v. 101, n. 6, p. 2414-2421, 2020.

WANG, Yan; WANG, Liuqing; LIU, Fei; WANG, Qi; SELVARAJ, Jonathan Nimal; XING, Fuguo; ZHAO, Yueju; LIU, Yang. Ochratoxin A producing fungi, biosynthetic pathway and regulatory mechanisms. Toxins, v. 8, n. 3, p. 1-15, 2016.

WELKE, Juliane Elisa. Fungal and mycotoxin problems in grape juice and wine industries. Current Opinion in Food Science, v. 29, p. 7-13, 2019. DOI 10.1016/j.cofs.2019.06.009.

ZHANG, Hongyin; GODANA, Esa Abiso; SUI, Yuan; YANG, Qiya; ZHANG, Xiaoyun; ZHAO, Lina. Biological control as an alternative to synthetic fungicides for the management of grey and blue mould diseases of table grapes: a review. Critical Reviews in Microbiology, v. 46, n. 4, p. 450-462, 2020.

ZHANG, Jian; ZHU, Liuyang; CHEN, Haoyu; LI, Min; ZHU, Xiaojuan; GAO, Qiang; WANG, Depei; ZHANG, Ying. A Polyketide Synthase Encoded by the Gene An15g07920 Is Involved in the Biosynthesis of Ochratoxin A in Aspergillus niger. [S. I.: s. n.], 2016. v. 64. 